\section{Detection of Epidemic Scarlet Fever Group A Streptococcus in Australia}

\begin{abstract}
Mark J. Walker, ${ }^{1, a}$ Stephan Brouwer, ${ }^{1}$ Brian M. Forde, ${ }^{1}$ Kate A. Worthing, ${ }^{2}$ Liam McIntyre, ${ }^{2}$ Lana Sundac, ${ }^{3}$ Sam Maloney, ${ }^{3}$ Leah W. Roberts, ${ }^{1}$ Timothy C. Barnett, ${ }^{1}$ Johanna Richter, ${ }^{1}$ Amanda J. Cork, ${ }^{1}$ Adam D. Irwin, ${ }^{4,5}$ Yuanhai You, ${ }^{6}$ Jianzhong Zhang, ${ }^{6}$ Gordon Dougan, ${ }^{7}$ Kwok Y. Yuen, ${ }^{8,9,10}$ Victor Nizet, ${ }^{11}$ Scott A. Beatson, ${ }^{1, a}$ Keith Grimwood, ${ }^{12,13, a}$ and Mark R. Davies ${ }^{2, a}$

${ }^{1}$ Australian Infectious Diseases Research Centre, University of Queensland, Brisbane, ${ }^{2}$ Department of Microbiology and Immunology, University of Melbourne, at the Peter Doherty Institute for Infection and Immunity, Victoria, Australia ${ }^{3}$ Pathology Queensland, Gold Coast Laboratory, and ${ }^{4}$ University of Queensland Centre for Clinical Research, and ${ }^{5}$ Queensland Children's Hospital, Brisbane, Australia; ${ }^{6}$ State Key Laboratory of Infectious Disease Prevention and Control, Collaborative Innovation, Center for Diagnosis and Treatment of Infectious Diseases, National Institute for Communicable Disease Control and Prevention, Chinese Center for Disease Control and Prevention, Beijing; ${ }^{7}$ Wellcome Trust Sanger Institute, Hinxton, Cambridge, United Kingdom; ${ }^{8}$ Department of Microbiology, ${ }^{9}$ Research Centre of Infection and Immunology, and ${ }^{10}$ State Key Laboratory for Emerging Infectious Diseases, University of Hong Kong, Hong Kong Special Administrative Region, China; ${ }^{11}$ Division of HostMicrobe Systems and Therapeutics, Department of Pediatrics, University of California, San Diego, La Jolla; and ${ }^{12}$ Menzies Health Institute Queensland, Griffith University, Gold Coast, and ${ }^{13}$ Departments of Infectious Diseases and Pediatrics, Gold Coast Health, Queensland, Australia
\end{abstract}

Sentinel hospital surveillance was instituted in Australia to detect the presence of pandemic group A Streptococcus strains causing scarlet fever. Genomic and phylogenetic analyses indicated the presence of an Australian GAS emm12 scarlet fever isolate related to United Kingdom outbreak strains. National surveillance to monitor this pandemic is recommended.

Keywords. Steptococcus pyogenes; scarlet fever; outbreak; superantigen.

Epidemic scarlet fever caused by the gram-positive bacterial pathogen Streptococcus pyogenes (group A Streptococcus [GAS]) resulted in significant childhood disease in the 19th and early 20th centuries, gradually abating in parallel with improved living conditions, enhanced standards of care, and the advent of antibiotics. By the beginning of this millennium, scarlet fever was considered a disease of negligible concern [1]. Yet, epidemic scarlet fever has been reported in Hong Kong [2, 3] and mainland China [4, 5] since 2011, and in the United Kingdom (UK) since 2014 [6-8] (Supplementary Figure 1). GAS isolates from these outbreaks have been multiclonal, encompassing several GAS emm types including emm1, emm12 (UK, Hong Kong,

Received 19 October 2018; editorial decision 25 January 2019; accepted 30 January 2019; published online February 5, 2019.

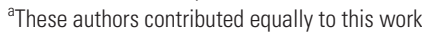

Correspondence: M. J. Walker, University of Queensland, School of Chemistry and Molecular Biosciences, Cooper Road, St Lucia, 0LD, 4072, Australia (mark.walker@uq.edu.au).

Clinical Infectious Diseases ${ }^{\circledR} \quad 2019 ; X X(X X): 1-3$

(C) The Author(s) 2019. Published by Oxford University Press for the Infectious Diseases Society of America. All rights reserved. For permissions, e-mail: journals.permissions@oup.com. DOl: 10.1093/cid/cizo99 mainland China), emm3, and emm4 (UK), and frequently encoding superantigens SpeA, SpeC, and SSA. Scarlet fever is a notifiable disease in Hong Kong, mainland China, and the UK. However, many national public health systems, including that of Australia, do not require scarlet fever case notification, making global tracking of epidemic strains intrinsically difficult. In 2016 we established a sentinel hospital surveillance (described in the Supplementary Information) to monitor the importation of GAS isolates causing epidemic scarlet fever into Australia. GAS isolates from 8 confirmed cases of scarlet fever (between 2016 and 2017) were selected for further analysis (Supplementary Figure 2; Supplementary Table 1; Supplementary Information). The GAS emm sequence type was determined and isolates were screened by polymerase chain reaction (PCR) for the presence of genes encoding superantigens ssa, speC, speA, and the erythromycin resistance gene $\operatorname{erm} B$ (recently associated with scarlet fever isolates from Hong Kong and mainland China) (described in the Supplementary Information). This preliminary PCR analysis identified the atypical presence of the ssa gene [9] in an Australian emm12 GAS isolate (designated SP1336) causing scarlet fever (Supplementary Figure 2C; Supplementary Table 1; Supplementary Information).

The complete genome sequence of SP1336 was determined using a multiplatform approach incorporating sequence data derived from short-read (Illumina), long-read (Pacific Bioscience and Oxford Nanopore), and PCR-derived capillary sequencing. This approach (described in the Supplementary Information) resolved ambiguity within the SP1336 genome due to the presence of multiple related prophages that affected the provisional genome assembly. After several rounds of assembly validation, we determined that SP1336 contained a 1878 827-bp circular genome (Figure 1; GenBank accession number CP031738).

Phylogenetic analysis of the $248 \mathrm{emm} 12$ genomes relative to the benchmark Hong Kong scarlet fever isolate HKU16 [2] revealed general geographical segregation of scarlet fever isolates from Hong Kong, mainland China, and the UK as reported previously (Figure 1C) [4]. SP1336 shared a close evolutionary relationship (17 core genome single-nucleotide polymorphisms) to a subclade of isolates from the UK that included clinical scarlet fever isolates. This clade did not share a recent evolutionary history with 2 other Australian emm 12 strains isolated from non-scarlet fever cases in the 1990s, indicating that they have evolved independently. Instead, these data suggest an evolutionary relationship between scarlet fever cases in the UK and this scarlet fever isolate from Australia.

One of the defining clinical features of scarlet fever is the cutaneous rash believed to be driven by potent immunostimulatory toxins such as the GAS streptococcal pyogenic exotoxins. 


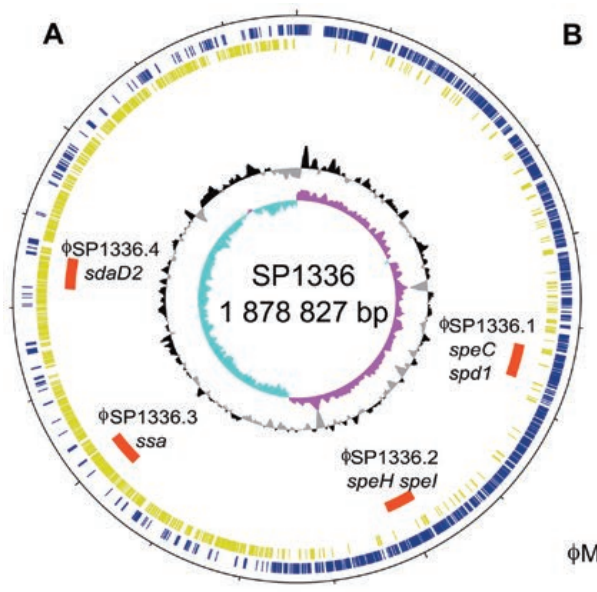

B

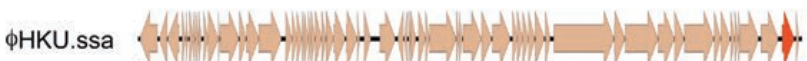

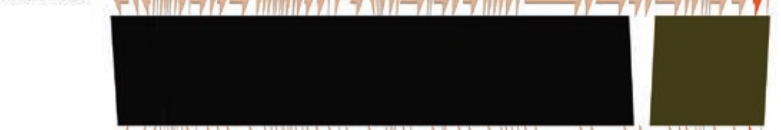

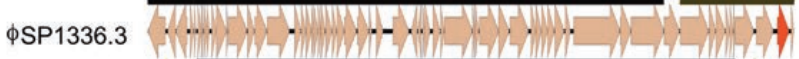

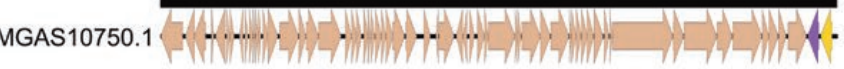

C

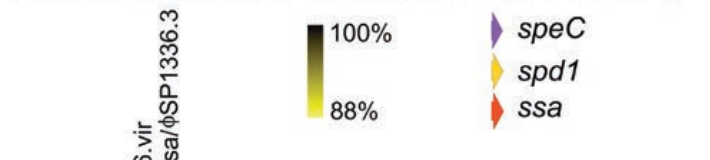

Country key

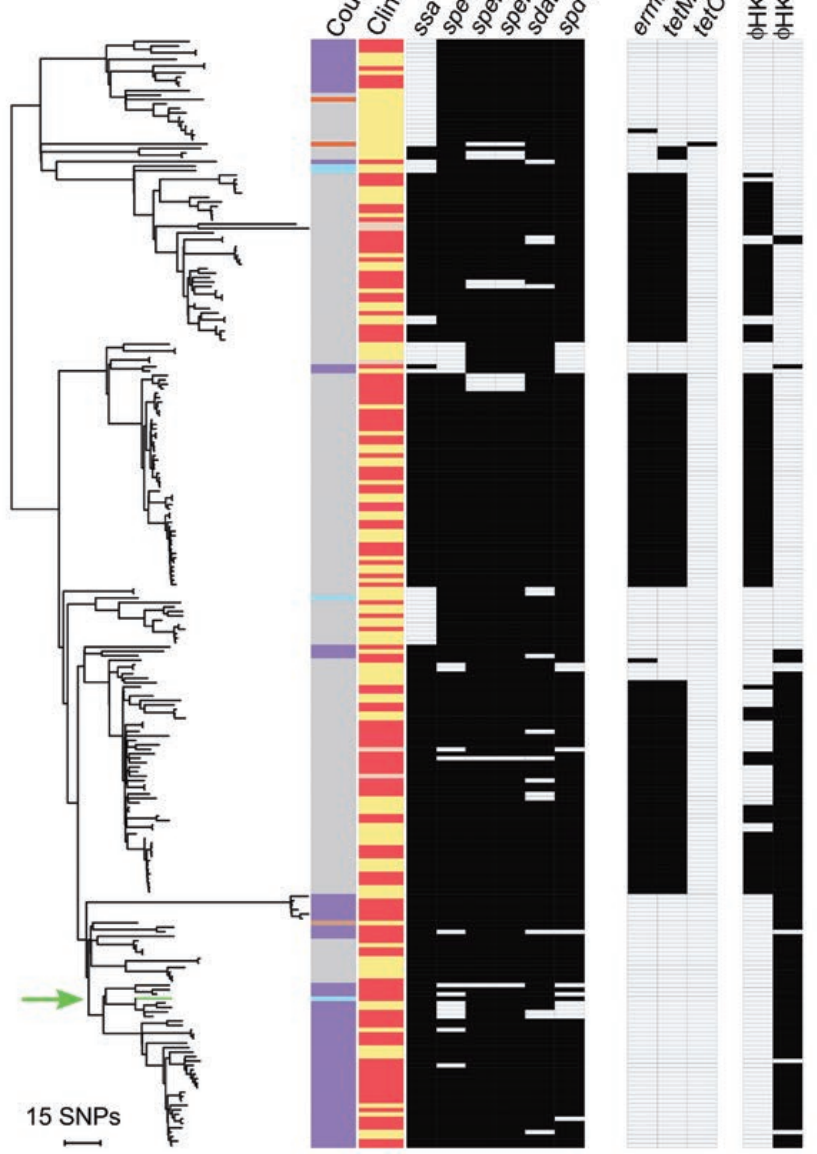

Australia

Americas

Lebanon

United Kingdom

Mainland China and Hong Kong

\section{Clinical key}

Clinical scarlet fever

Non-scarlet fever

Clinically undefined

\section{Antigen screen key}

Presence

Absence

Figure 1. Population genomics of the Australia scarlet fever strain SP1336. A, Genome ring of the SP1336 emm12 group A Streptococcus (GAS) genome showing (from inner ring); GC skew, GC plot, position and name of prophage elements (red blocks), and location and orientation of coding sequences. B, Genomic organization of $\Phi S P 1336.3$ and $\Phi S P 1336.1$ relative to their closest genetic relatives; $\Phi$ HKU.ssa (from an emm12 Hong Kong scarlet fever strain HKU360 [3]) and $\Phi M G A S 10750$ (from an emm4 pharyngitis isolate from the United States [10]), respectively. Nucleotide sequence diversity is scaled from $100 \%$ (black) to $80 \%$ (yellow). C, Midpoint-rooted maximum-likelihood phylogenetic tree of $248 \mathrm{emm} 12 \mathrm{GAS}$ strains built on 2633 polymorphic sites within the nonrecombinogenic core genome relative to the HKU16 reference isolate [2]. Clinical association and country of isolation are displayed next to the tree in addition to the relative distribution of selected virulence genes, and antimicrobial resistance genes and ssa-carrying prophages (ФHKU.vir [2], $\Phi H K U . s s a ~[3]$, and $\Phi S P 1336.3$ ) within the emm12 population (black shading refers to gene carriage). Phylogenetic branch relating to the Australian scarlet fever isolate SP1336 is colored in green and indicated by an arrow for visual aid. Abbreviation: SNP, single-nucleotide polymorphism. 
The emergence of major scarlet fever clades in China has been linked to the acquisition of SSA-, SpeC-, and Spd1-encoding prophages [3]. Analysis of the prophage content of the SP1336 genome revealed 4 prophage elements designated ФSP1336.1 (harboring the exotoxin SpeC and the DNase, Spd1), ФSP1336.2 (toxins SpeH and SpeI), ФSP1336.3 (superantigen SSA), and ФSP1336.4 (streptodornase, SdaD2) (Figure 1A). This emm12 prophage profile is similar to that found in the ongoing scarlet fever outbreaks in the UK, Hong Kong, and mainland China [3, 4]. Comparative analyses of the $\Phi S P 1336.3$ and other ssa-harboring prophages from Hong Kong and mainland China showed that $\Phi S P 1336.3$ is approximately 95\% identical at the nucleotide level to the ssa prophage $\Phi H K U . s s a$ from the Hong Kong scarlet fever strain HKU360 (Figure 1B) [3]. ФSP1336.3 also shared a high degree of synteny with ФSP1336.1 ( 90\%), itself approximately $99 \%$ nucleotide identity to prophage QMGAS10750.1 from a pharyngitis patient in the United States (Figure 1B) [10]. No multidrug resistance genes or transposable elements were identified in the SP1336 isolate, commensurate with the evolutionary-related emm 12 UK lineage.

Scarlet fever cases have risen in multiple countries since 2011 (Supplementary Figure 1) [2-8]. Rather than subsiding, case numbers of scarlet fever have again significantly increased in recent years both in Hong Kong (Supplementary Figure 1A) and the UK [11]. The burden of this outbreak on healthcare services is substantial with hospital admissions from scarlet fever increasing 3 -fold from baseline at the peak of the outbreak [8]. We have become increasingly concerned about the spread of scarlet fever-inducing GAS to countries where this disease is not notifiable, and thus instigated our own localized notification system in Australia. Here we report the detection of an emm12 GAS strain in Australia that harbors scarlet fever-associated phagelike elements $[3,4]$ and shares a recent common ancestor to a cluster of scarlet fever isolates from the UK. The presented data highlight the dynamic nature of toxin-harboring prophages within polylysogenic GAS genomes. Furthermore, the maintenance of distinct virulence gene sets (SSA, SpeC, Spd1, SpeH, SpeI) suggests a positive selection for particular toxin combinations within the current scarlet fever pandemic.

Outbreaks of scarlet fever in the UK, Hong Kong, and mainland China have been characterized as multiclonal, encompassing multiple emm types [2-4, 6-8]. While northern Asian and UK emm12 scarlet fever isolates are distantly related, these geographically discrete strains have evolved independently into distinct clades [3,4], excluding the worldwide spread of a single emm12 scarlet fever clone. GAS superantigens SpeA, SpeC, and SSA have been associated with scarlet fever isolates in several studies, but this association is not universal $[2-4,6]$. The underlying cause of disease resurgence remains unknown. Immune status changes in the human population resulting in increased susceptibility to infection, introduction of genetic elements encoding superantigens into the GAS population, coinfection with an unknown agent that predisposes the host to scarlet fever, and environmental change have all been proposed as possible triggers for the observed resurgence in disease [12]. While a single dominant strain fails to explain the recent international upsurge in scarlet fever, the distant identification of a strain associated with the UK outbreak may herald similar outbreaks elsewhere. In national health systems where scarlet fever is not notifiable, sentinel hospital surveillance of the type used in this study to rapidly identify and monitor the dissemination of GAS isolates causing epidemic scarlet fever is warranted. Such surveillance would underpin public health interventions aimed at limiting further propagation.

\section{Supplementary Data}

Supplementary materials are available at Clinical Infectious Diseases online. Consisting of data provided by the authors to benefit the reader, the posted materials are not copyedited and are the sole responsibility of the authors, so questions or comments should be addressed to the corresponding author.

\section{Notes}

Acknowledgments. The authors thank Dr Shane George, who provided information from the Emergency Department Information System.

Financial support. This work was supported by the National Health and Medical Research Council of Australia; the Wellcome Trust, UK; the Chinese Center for Disease Control and Prevention, People's Republic of China; the Department of Health Consultancy Service for Enhancing Laboratory Surveillance of Emerging Infectious Diseases, Hong Kong; Research Capability on Antimicrobial Resistance, Hong Kong; and the Queen Mary Hospital Charitable Trust, Hong Kong.

Potential conflicts of interest. All authors: No reported conflicts. All authors have submitted the ICMJE Form for Disclosure of Potential Conflicts of Interest. Conflicts that the editors consider relevant to the content of the manuscript have been disclosed.

\section{References}

1. Morens DM, Folkers GK, Fauci AS. The challenge of emerging and re-emerging infectious diseases. Nature 2004; 430:242-9.

2. Tse H, Bao JYJ, Davies MR, et al. Molecular characterization of the 2011 Hong Kong scarlet fever outbreak. J Infect Dis 2012; 206:341-51.

3. Davies MR, Holden MT, Coupland P, et al. Emergence of scarlet fever Streptococcus pyogenes emm 12 clones in Hong Kong is associated with toxin acquisition and multidrug resistance. Nat Genet 2015; 47:84-7.

4. You Y, Davies MR, Protani M, McIntyre L, Walker MJ, Zhang J. Scarlet fever epidemic in China caused by Streptococcus pyogenes serotype M12: epidemiologic and molecular analysis. EBioMedicine 2018; 28:128-35.

5. Liu Y, Chan TC, Yap LW, et al. Resurgence of scarlet fever in China: a 13-year population-based surveillance study. Lancet Infect Dis 2018; 18:903-12.

6. Turner CE, Pyzio M, Song B, et al. Scarlet fever upsurge in England and molecular-genetic analysis in north-west London, 2014. Emerg Infect Dis 2016; 22:1075-8.

7. Chalker V, Jironkin A, Coelho J, et al. Genome analysis following a national increase in scarlet fever in England 2014. BMC Genomics 2017; 18:224.

8. Lamagni T, Guy R, Chand M, et al. Resurgence of scarlet fever in England, 201416: a population-based surveillance study. Lancet Infect Dis 2018; 18:180-7.

9. Commons R, Rogers S, Gooding T, et al. Superantigen genes in group A streptococcal isolates and their relationship with emm types. J Med Microbiol 2008; 57:1238-46.

10. Beres SB, Musser JM. Contribution of exogenous genetic elements to the group A Streptococcus metagenome. PLoS One 2007; 2:e800.

11. Public Health England. Group A streptococcal infections: third report on seasonal activity, 2017 to 2018. Health protection report. London, UK: Public Health England, 2018:12.

12. Walker MJ, Brouwer S. Scarlet fever makes a comeback. Lancet Infect Dis 2018 18:128-9. 